\title{
SPALLATION NEUTRON SOURCE LINAC VACUUM SEAL DESIGN AND TESTING*
}

\author{
Z. Chen ${ }^{\dagger}$, C. Gautier, and N. Bultman, LANL, Los Alamos, NM 87545, USA
}

\begin{abstract}
Optimum seal selection with respect to vacuum performance, RF electrical performance, life, cost, reliability, and ease of installation is of primary importance. This report summarizes an investigation of metallic vacuum seals including the $\mathrm{C}$-seal, energized spring seal, Helicoflex copper delta seal, aluminum delta seal, delta seal with limiting ring, and a prototype copper diamond seal. This study was conducted to support the Drift Tubing Linac (DTL) and Coupled Cavity Linac (CCL) design efforts for the Spallation Neutron Source (SNS) project. A series of vacuum leak rate tests using necessary reduced thickness flange designs has been completed. Copper plated stainless steel flanges, necessary for flanged joints intersecting RF cavities, have also been studied and tested. Detailed structural modeling of the CCL bridge coupler seal and flange has also been completed to verify flange structural integrity and appropriate seal compressive loading.
\end{abstract}

\section{INTRODUCTION}

Vacuum seals are very important for ensuring that the Spallation Neutron Source (SNS) Linac has an optimum vacuum system. The vacuum joints between flanges must have reliable seals to minimize the leak rate in order to meet vacuum and electrical requirements. The seals should also be easy to install and to replace for maintenance purposes. In this report, the vacuum seal selection and testing for beam line and bridge coupler of the Coupled Cavity Linac (CCL) will be described.

A vacuum seal is desired that requires significantly less loading than a copper gasket standard with ConFlat and good electrical conductivity for RF coupling cavities. A vacuum seal is also required at the beam line, where various hardware items have to be installed. The thinner flanges are utilized in the design. The leak rate and torque applied to the thin ConFlat flange with a copper gasket are a baseline for the vacuum test on all seals and thin flanges. We have prototypes of 3.38 thin ConFlat flange with a copper gasket for beamline vacuum joints. The tests were completed by VARIAN. An estimated sealing load is too high at 4,173 pounds per inch of sealing circumference for the beamline flanges [1]. On the other hand, the knife-edges of ConFlat flanges lose their sealing function after being exposed to $450^{\circ} \mathrm{C}$ [2]. Furnace brazing of the accelerating cavities requires temperatures far in excess of $450^{\circ} \mathrm{c}$. Apparently, the conflat flange and copper gasket cannot be used in the

* Work supported by the Office of Science, Basic Energy Sciences, of the U.S. Department of Energy; and by Oak Ridge National Laboratory. † zchen@lanl.gov
SNS CCL beam line and bridge couplers

\section{METAL SEALS DESCRIPTION}

The seal locations are shown in a typical CCL intersegment as in Figure 1.

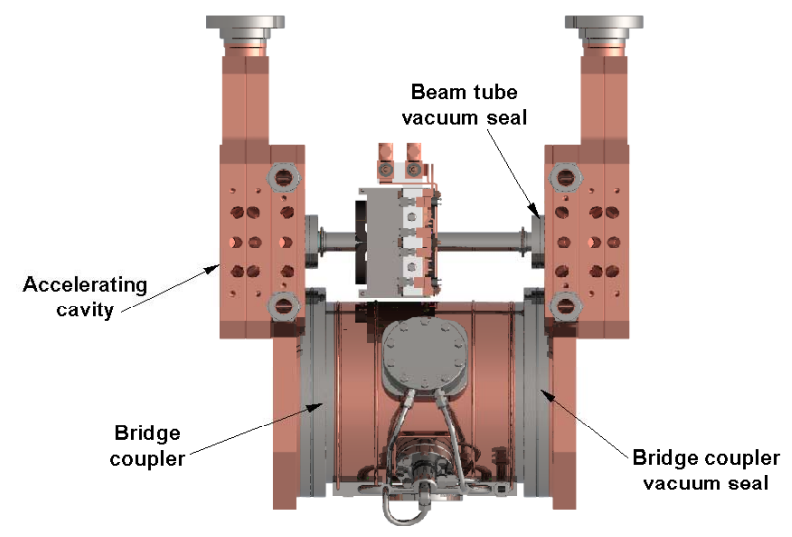

Figure 1: Typical CCL Inter-segment

In order to meet the requirements of vacuum, RF electric current continuity, installation, and structural design, the silver coated metal C-seal, Inconel reinforced spring C-rings, Helicoflex delta seal jacketed with copper or aluminum $(\mathrm{Cu}$ delta or $\mathrm{Al}$ delta, hereafter), using stainless steel 300 series or Inconel alloy 600 liner, and Inconel reinforced spring were selected in an R \& D study on leak rate test. A special effort was made to produce a prototype of a copper diamond seal referencing the aluminum diamond seal that was used in the beamline at CERN. The cross-section has the "diamond edges" for the sealing surface area. Two prototypes of the copper diamond seal have also been tested.

To minimum the leak paths that are determined by the profiles of the surfaces in contact [3], all sealing faces (grooved face and cover) were machined to a maximum $\mathrm{Ra}$ roughness of $0.8 \mu \mathrm{m}$. The hardness of flange surface for copper delta seal is 120 Vickers, and 65 Vickers for aluminum delta seal.

The testing has two types of the flange surface options. The flanges are made of stainless steel. We tested the copper delta seals with and without copper plated surface. Tests are conducted to determine if the copper delta seal could be used for the softer sealing surface of copper coated stainless steel flanges. The flange geometry has also two options. One option has a groove at the sealing face, another one is simple flat and positioned by the bolt pattern. 


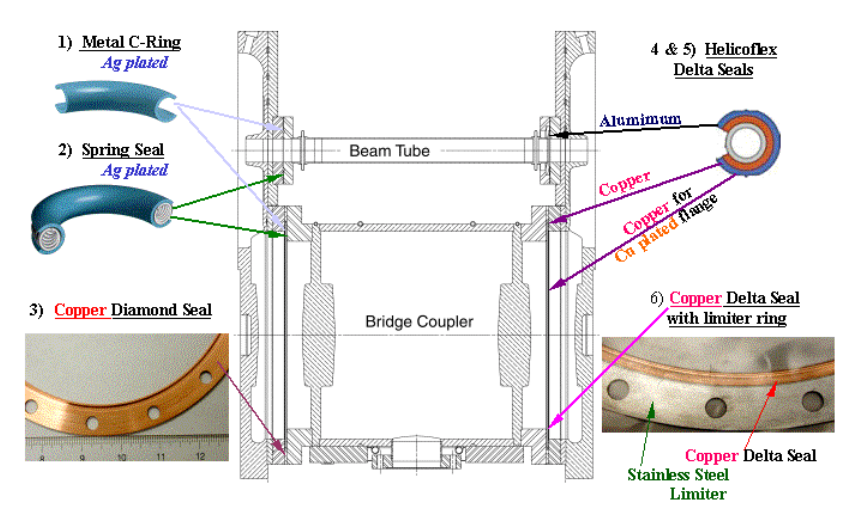

Figure 2: Overview of metal seal testing

\section{TESTING AND RESULTS}

To determine the leak rate and appropriate fastening torque we use the standard knife-edge flange with the copper gaskets seal as the baseline, and adopted ConFlat flange tightening sequence. The test hardware was made as a part of coupling cavity and a partial beam line. A Balzers QualyTest Helium leak Detector, which has lowest detectable leak rate of $5 \mathrm{E}-12 \mathrm{mbar}-1 / \mathrm{s}$, was utilized. All leak rate tests were performed using helium fill to the plastic envelope that bagged the testing hardware with the seal in the place. The leak rate is taken after helium filling lasts 3 minutes at least [4].

Seals are tested for bridge coupler vacuum joints using 8.8-in. OD flanges. There are four types of metal seals, including metal C-seal, spring C-ring, copper delta seal and copper diamond seals. Figure 4 shows the leak rate corresponding to the torque applied level for two prototypes of the diamond seal, and Figure 3 shows the leak rate of others.

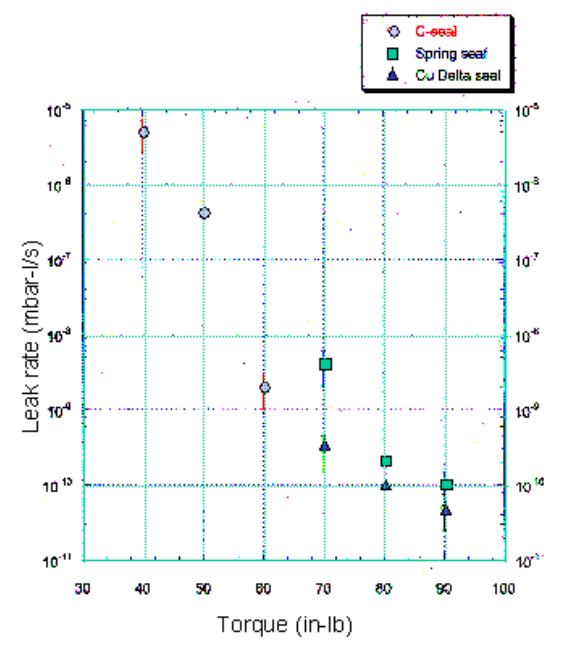

Figure 3: Leak rate of C-seal, Spring C-ring, and

Copper delta seal for coupling cavity

We use 3.38 in. OD flanges and some small sized seals for the beam line vacuum joints leak rate testing. Compared with the copper delta seal for the coupling cavity, we use an aluminum delta seal for the beam line flange, because it does not require any electric conductivity, while it is very important for coupling cavity connections. The test results are plotted in Figure 5.

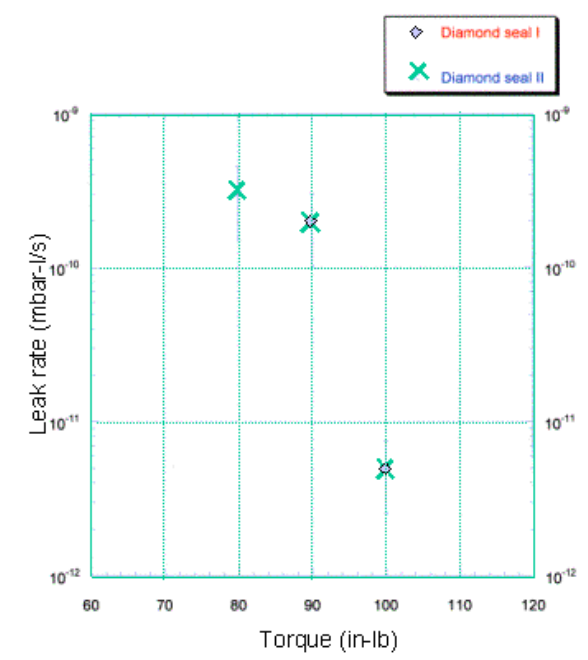

Figure 4: Leak rate of copper diamond seal for coupling cavity vacuum sealing

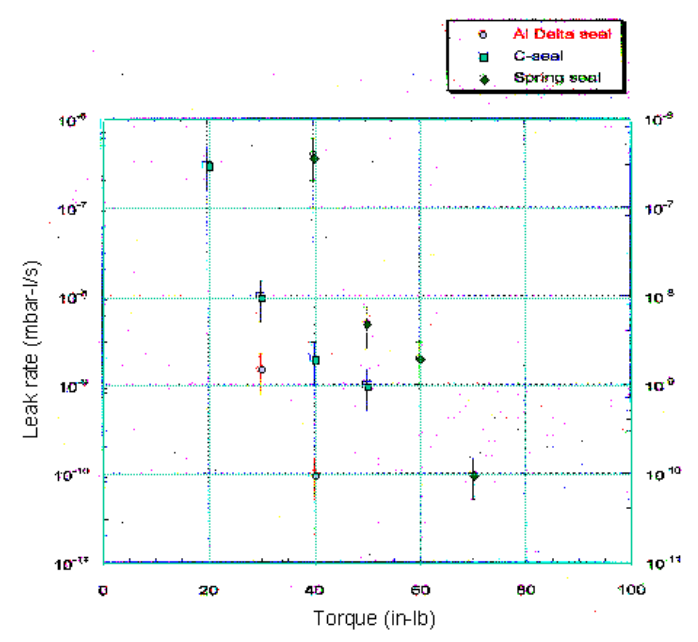

Figure 5: Leak rate of tested seals for beam line Vacuum sealing

The copper delta seal requests that the sealing face must have appropriate hardness. The tested flange is made of stainless steel. Considered the flanges of the bridge coupler have to have copper plated. To ensure the copper delta seal still matches the leak rate requirement when the stainless steel flange has copper plated, the leak rate test for this case is also performed. We have two seals to complete this test. After the first seal tested, a clear footprint of the delta seal occurred at the copper plated surface. The photo is shown in Figure 6. The footprint did not change much after the second seal was tested. The leak rate results from two seals are very close. A comparison chart of the leak rate test on copper delta seal with and without copper plated flange is shown in Figure 7. 


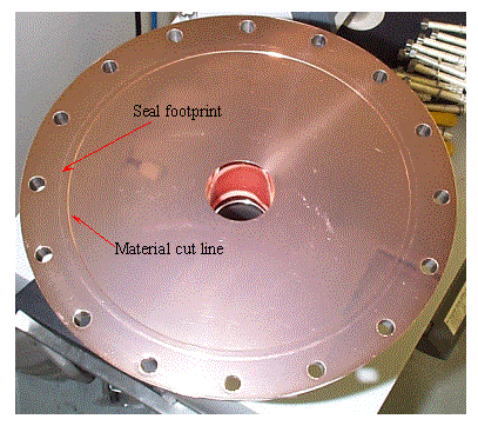

Figure 6: A photo of copper plated flange after vacuum leak rate test with copper delta seal

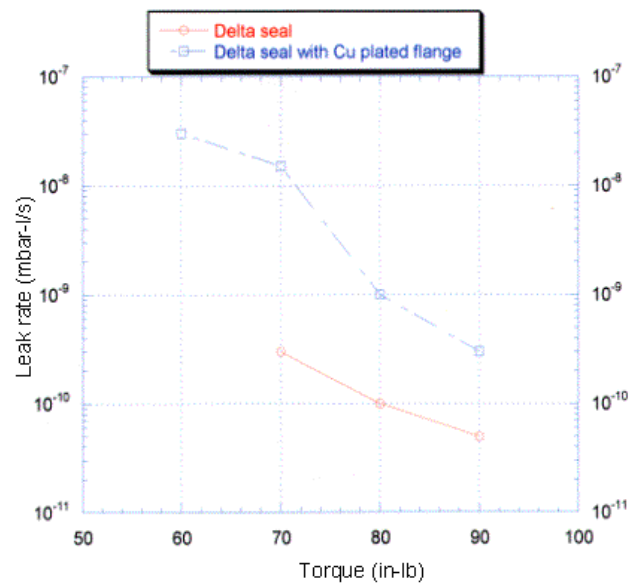

Figure 7: Comparison chart of leak rate test on copper delta seal with and without copper plated flange

All the test results have helped us determine the suitable seals that are reliable for SNS bridge couplers and beamline joints as compared with ConFlat flanges and copper gasket joints.

Both copper delta seal and copper diamond seal have excellent sealing performance. Considering the cost and ease of installation, a copper delta seal with a stainless steel limiter ring was developed. The seating load is 1,050 lbs per circumference inch of seal. The stainless steel limiter ring controls the compressed limit of the copper delta seal. Even though there is $>85 \%$ probability of the delta seal being reusable a second time [5], it is still risky to reuse the seal for Linac maintenance. Another advantage of the copper delta seal with limiter ring is that the limiter ring is reusable. It not only simplifies the installation procedure, but also reduces the maintenance cost by using a refurbished seal.

\section{CONCLUSIONS}

A vacuum leak rate test on several types of metal seals has been completed at Los Alamos Laboratory. Testing was performed using a Balzers QualyTest ${ }^{\mathrm{TM}}$ Helium Leak detector. A standardized condition of 1 atmosphere differential pressure at room temperature was used in all cases. The test results of leak rate on the purchased seals have matched the manufacturers' published values. Most of these seals have the capability of achieving the required CCL vacuum level of less than 5E-8 torr, except the metal $\mathrm{C}$-seals. The leak rate of metal $\mathrm{C}$-seals is marginal. The lowest leak rate of $5 \mathrm{E}-12 \mathrm{mbar}-1 / \mathrm{s}$ was achieved by two prototypes of the copper diamond seals.

In addition to matching vacuum and RF requirements, other seal selection criteria considered reliability, seating load, risk assessment, ease of installation, and replacement. The Helicoflex delta seals are best suitable for use in the vacuum joints at the beam line and long bridge coupling cavities of the SNS warm linac. A leak rate of the aluminum delta seals for beam line joints is $1 \mathrm{E}-$ $10 \mathrm{mbar}-1 / \mathrm{s}$ under a torque of $40 \mathrm{in}$. $-\mathrm{lb}$, and a leak rate of the copper delta seals for the bridge coupler is $5 \mathrm{E}-11$ mbar-1/s under $90 \mathrm{in}$. $-\mathrm{lb}$. Since this design needs to add a groove on the bridge coupling cavity flange, a delta seal with a pressurized limited ring is selected, which has the advantage of a diamond seal and is more reliable for the vacuum joints of SNS bridge coupling cavities. The results from both seal tests with copper plated flange were very close in the level of $5 \mathrm{E}-10 \mathrm{mbar}-1 / \mathrm{s}$ which was one order higher than the result from the delta seal test with non-copper plated flange under the same torque of $90 \mathrm{in.-}$ $\mathrm{lb}$. Even though the softer copper plated layer has increased the leak rate, it still matches the requirement that a leak rate be less than $8 \mathrm{E}-11$ torr- $1 / \mathrm{s} / \mathrm{mm}$ with a seal perimeter of over $1,000 \mathrm{~mm}$.

\section{REFERENCES}

[1] S. Goff, N. Mills, VARIAN on "Los Alamos Thin ConFlat Flange Sealing Information" report, (1999).

[2] MDC Vacuum Products cooperation p17. (1992).

[3] A. Roth, Vacuum Technology, 7.3(395), 1982.

[4] Z. Chen, C. Gautier, F. Hemez, and N. Bultman, LA-13698-MS, Los Alamos National Lab, (2000).

[5] Welch, K. et al Metal and Elastomer Seal Tests for Accelerator Application, BNL, (1990). 